\title{
Un modelo biogeoquímico para el Golfo de Nicoya, Costa Rica
}

\author{
Farid A. Tabash Blanco \\ Estación de Biología Marina, Escuela de Ciencias Biológicas, Universidad Nacional. Puntarenas, Costa Rica. \\ Apartado Postal: 86-3000; atabash@una.ac.cr
}

Recibido 29-VII-2004. Corregido 20-VI-2006. Aceptado 15-XII-2006.

\begin{abstract}
A biogeochemical model for the Gulf of Nicoya, Costa Rica. In agreement with the Broecker and Penn two-boxes model, I generated a biogeochemical balance model for the Gulf of Nicoya (Guanacaste, Costa Rica) using two nutrient reservoirs: surface water and deep water. The mixing zone was located at a depth of $20 \mathrm{~m}$. There is a balance between surface waters descending to the bottom and upwelling waters that carry nutrients and other chemical elements to the surface. The main source of nitrogen (nitrate), was the outlet of the Tempisque and Tárcoles rivers. The Gulf of Nicoya is a net source of Dissolved Inorganic Nitrogen (DIN) with an availability rate of $87 \times 10^{3} \mathrm{~mol} \mathrm{day}^{-1}$ in the dry season and $3044 \times 10^{3} \mathrm{~mol} \mathrm{day}^{-1}$ in the rainy season. Dissolved Inorganic Phosphate (DIP) was estimated in 27 mol day $^{-1}$ in the dry season and 207 mol day- ${ }^{1}$ in the rainy season. The dynamics of these biolimited nutrients, in relation to runoff seasonal variations, fits the biological processes reported for the gulf, for example, for variations in primary productivity levels, and maturity and reproduction seasons for species with short and long life cycles. Rev. Biol. Trop. 55 (1): 33-42. Epub 2007 March. 31.
\end{abstract}

Key words: tropical, estuary, nutrients, biogeochemical model, Nicoya Gulf, Costa Rica.

La dinámica de los sistemas marinos se ha convertido en un paradigma; el descubrimiento de su complejidad y codificar esta dinámica se considerada como una manera adecuada de analizar los sistemas marinos, ello con el propósito de comprenderlos, intentar simularlos y de esta forma lograr algún grado de predicción de la conducta del sistema (Huwe y van der Ploeg 1992).

Los modelos biogeoquímicos tratan de explicar los flujos de material dentro de un sistema, lo cual puede efectuarse por una gran variedad de vías. Esencialmente, se cuantifican los ingresos de materia orgánica e inorgánica, que son aportados hacia un sistema dado, la tasa en que estos materiales se transforman, son utilizados o removidos dentro de este sistema y la cantidad de materia involucrada en estos procesos (Schlitzer 2000).
Epifanio et al. (1983), Krees et al. (1997) y Calvo (2002) afirman que en el Golfo de Nicoya el aporte de elementos biolimitantes como el nitrógeno y el fósforo son principalmente de tipo alóctono y su abundancia sufre variaciones estacionales, especialmente en la parte interna, consecuencia del aporte de los ríos entre las épocas seca y lluviosa. Valdes et al. (1987) afirma que las mayores concentraciones de nitrógeno y fósforo se presentan durante la época seca; mientras que Córdoba-Muñoz (1993) no encontró diferencias estacionales significativas en sus concentraciones.

El modelo biogeoquímico propuesto es producto del cálculo de balances de masas de variables específicas (agua, sedimentos, nitrógeno, fósforo, etc.) en un área geográfica y durante un periodo de tiempo definido que representa los procesos verticales que se 
encuentran en el Golfo de Nicoya. Para ello se considera la resuspensión del sedimento, surgencias, procesos de mezcla vertical, aportes de ríos y el atmosférico; se evalúa la importancia de estos procesos en relación con los nutrimentos (ingreso o reciclamiento) y se consideran sólo dos reservorios de nutrimentos.

\section{MATERIALES Y MÉTODOS}

Para el desarrollo de este modelo de balance biogeoquímico del Golfo de Nicoya, se consideraron dos reservorios de nutrimentos: aguas superficiales y aguas profundas. La zona de mezcla se ubicó en promedio a los $20 \mathrm{~m}$ de profundidad y se considera que existe un balance entre las aguas superficiales que van hacia el fondo y un sistema de surgencia que lleva nutrimentos y otros elementos químicos del fondo a la superficie.

De acuerdo con el modelo Broecker y Peng (1982) se asume: (i) que la única vía por la que ingresan los elementos químicos al Golfo de Nicoya es por lixiviación desde el continente, a través de aportes fluviales, y (ii) que estos elementos aportados al mar son utilizados por medio de procesos biológicos. Estas partículas durante su caída a través de la columna de agua pueden ser utilizadas por predación, incorporación o remoción. De forma que tan sólo una pequeña proporción llega a sedimentar en el fondo, (iii) que este sistema se encuentra en equilibrio, ello porque los aportes de elementos químicos son iguales a las salidas o pérdidas, (iv) que la cantidad de agua que se hunde normalmente es mucho mayor que el agua que llega del fondo a través de surgencias y (v) que el ciclo de mezcla vertical de los constituyentes (Tm) en el Golfo de Nicoya se estima en 75 años. Para ello se consideraron datos informados por Peterson (1958), Voorhis et al. (1983), Brugnoli y Morales (1999) y Brenes et al. (2003) para calcularlo a partir de la relación Tm/g·f (Broecker y Peng 1982), donde $\mathrm{g}=$ fracción de la concentración de un constituyente " $x$ " que alcanza la superficie $y$ que es removido del fondo en forma particulada $\mathrm{y}$ “ $\mathrm{f}$ " representa la fracción de partículas que no son disueltas y que sedimentan. Dichos valores fueron promediados con informes locales y regionales de tiempos medios de residencia de constituyentes dentro de la bomba biológica en el océano (Sarmiento y Toggweiler 1984, Orr y Sarmiento 1992, Ganadesikan 1999, Archer et al 2001).

El modelo biogeoquímico planteado en esta propuesta es afectado también por los patrones de lluvias. En el Golfo de Nicoya, se presentan dos épocas climáticas, la época seca que va desde Enero hasta Mayo y la época lluviosa entre Junio y Diciembre. Las precipitaciones son aproximadamente de $50 \mathrm{~mm}$ por mes durante la época seca y más de $600 \mathrm{~mm}$ por mes en la época lluviosa, lo que representa una enorme variación estacional de los flujos de agua de escorrentía que recibe el Golfo de Nicoya.

Este incremento en los aportes de ambos ríos llega a su máximo de descarga durante la época lluviosa, en un ámbito de $40 \mathrm{a} 60 \mathrm{~m}^{3} \mathrm{~s}^{-1}$. La salinidad promedio del estuario llega a fluctuar entre 25 y 33 \%o (Epifanio et al. 1983). La concentración de Nitrógeno Inorgánico Disuelto (NID) incrementa hasta $3058 \times 103$ mol día ${ }^{-1}$, pero los niveles de concentración de fósforo se mantienen solo marginalmente afectados (Smith y Crossland 1999).

El modelo integra las variaciones en los aportes de agua proveniente de los ríos, de su efecto sobre la salinidad, de las variaciones del NID y de Fósforo Inorgánico Disuelto (FID), tanto para la época seca como la lluviosa, con el objeto de poder luego analizar sus variaciones anuales. Basado en esto se considera: (i) que el volumen de la escorrentía asociada con el flujo de agua de los ríos (Vr) se deposita completamente en la capa superficial del Golfo de Nicoya, lo que a su vez provoca, una variación en la salinidad superficial del sistema, (ii) que el flujo de agua oceánica que ingresa al Golfo de Nicoya proviene de la corriente ecuatorial subsuperficial (CESS) y (iii) que el flujo combinado proveniente de la superficie y del fondo se mezclan $(\mathrm{Vm})$. El balance salino del sistema se mantiene gracias a estos procesos de mezcla de aguas y a los procesos de surgencia 
de agua que transportan materiales desde el fondo a la superficie.

\section{RESULTADOS}

Modelo para los aportes de agua de los ríos y de las variaciones en salinidad en el Golfo de Nicoya: Los cálculos de los ingresos de agua de parte de los ríos Tempisque y Tárcoles y de las correspondientes variaciones en salinidad se obtuvieron considerando los niveles promedio de precipitación y de variación en salinidad obtenidos durante los cinco meses de época seca y los siete meses de la época lluviosa para el año 2001 (Fig. 1). La evaporación se asume como constante a una tasa de $1000 \mathrm{~mm}$ año-1, la cual se obtuvo considerando datos promedio de evaporación (Instituto Meteorológico Nacional 2001). El aporte de los ríos Tempisque y Tárcoles se estimó a partir de las mediciones de flujo informadas durante el 2001 por el Servicio Nacional de Riego y Avenamiento (SENARA) (Cuadro 1).

Otras estimaciones de importancia:

Volumen total del Golfo de Nicoya $=5500 \mathrm{x}$ $10^{6} \mathrm{~m}^{3}$

Cantidad de sales que ingresa al Golfo de Nicoya $=33.25 \%$ × $10^{3} \mathrm{~m}^{3} \mathrm{dí}^{-1}$

Evaporación neta promedio anual $=1000 \mathrm{~mm}$ año ${ }^{-1}$

Niveles de precipitación: época seca $=900 \mathrm{~mm}$ año-1

Época lluviosa $=10500 \mathrm{~mm}$ año-1

El Golfo de Nicoya incrementa su dinámica durante la época lluviosa, debido al aumento en los niveles de precipitación y al nivel de escorrentía proveniente de ambos ríos, provocando que los niveles de transporte de agua sean mayores y los tiempos de residencia menores.

En el Cuadro 2 se observan los resultados para la dinámica de flujos de agua determinados con este modelo del Golfo de Nicoya, tanto para la época seca como la lluviosa. Durante la época seca, el $86 \%$ del $\mathrm{NaCl}$ que alcanza la

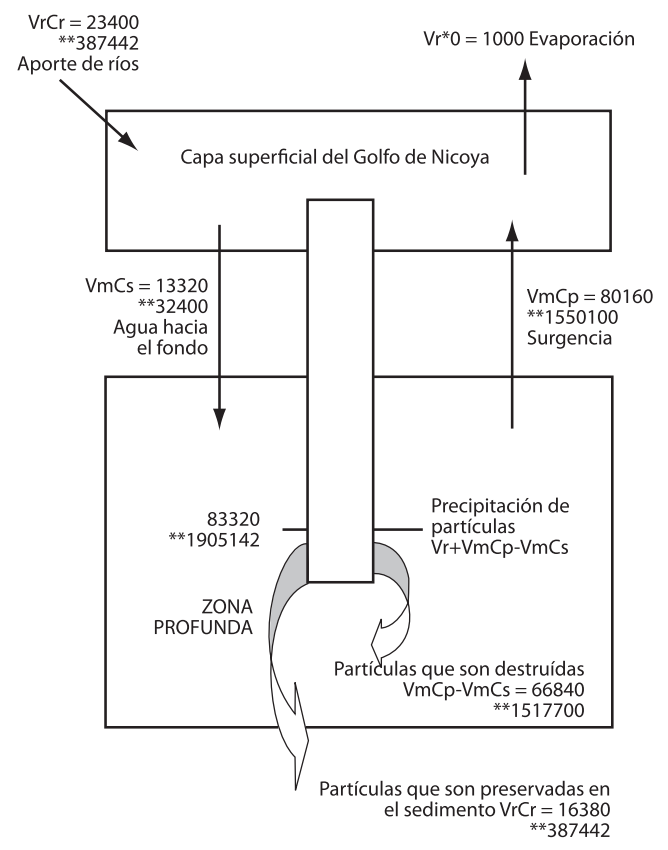

Fig. 1. Modelo de Broecker y Penn presentado para la relación entre los flujos de agua y los ingresos de $\mathrm{NaCl}$ en el Golfo de Nicoya, durante la época seca y la época lluviosa (**). Los flujos de agua se expresan en $10^{3} \mathrm{~m}^{3} \mathrm{dí}^{-1}$ y los de $\mathrm{NaCl}$ en $10^{3}$ psu $\mathrm{m}^{3} \mathrm{día}^{-1}$

Fig. 1. Broecker \& Penn model for the water flow and $\mathrm{NaCl}$ input relationship in the Gulf of Nicoya, during the dry and rainy (**) seasons. Water input in $10^{3} \mathrm{~m}^{3} \mathrm{day}^{-1}$ and $\mathrm{NaCl}$ in $10^{3}$ psu m $^{3}$ day $^{-1}$

\section{CUADRO 1}

Cuantificación de los parámetros de ingreso para la construcción del modelo biogeoquímico acorde con Broecker y Penn (1982)

\section{TABLE 1}

Input parameters quantified forBroecker \& Penn's biogeochemical model

\begin{tabular}{|c|c|c|}
\hline Parámetro & época seca & época lluviosa \\
\hline $\operatorname{Vr}\left(10^{3} \mathrm{~m}^{3} \mathrm{dí}^{-1}\right) * *$ & 1675 & 25300 \\
\hline $\operatorname{Cr}\left(\right.$ psu $\left.\times 10^{3} \mathrm{~m}^{3} \mathrm{dí}^{-1}\right) *$ & 23.4 & 32.02 \\
\hline $\operatorname{Vm}\left(10^{3} \mathrm{~m}^{3} \mathrm{dia}^{-1}\right)^{*}$ & 1200 & 3000 \\
\hline $\mathrm{Cp}\left(\text { psu } \times 10^{3} \mathrm{~m}^{3} \mathrm{dí}^{-1}\right)^{*}$ & 66.8 & 516.7 \\
\hline Cs $\left(\mathrm{psu} \times 10^{3} \mathrm{~m}^{3} \mathrm{di}^{\prime} \mathrm{a}^{-1}\right)^{*}$ & 11.1 & 10.8 \\
\hline
\end{tabular}

- Smith y Crossland(1999). ** datos obtenidos de registros de mediciones mensuales del SENARA, referidos al promedio obtenido para el año 2001. 
CUADRO 2

Dinámica de flujos de agua estimados por el modelo de Broecker y Penn para el Golfo de Nicoya, durante la época seca y la lluviosa

TABLE 2

Input of water flows estimated by Broecker \& Penn model's for the Gulf of Nicoya, during the dry and rainy seasons

\begin{tabular}{lcc}
\multicolumn{1}{c}{ Parámetro } & época seca & época lluviosa \\
$\mathrm{G}$ & 0.86 & 0.98 \\
$\mathrm{Cp} / \mathrm{Cr}\left(10^{3} \mathrm{psu} \mathrm{m}^{3} \mathrm{día}^{-1}\right)$ & 2.85 & 16.1 \\
$\mathrm{Cs} / \mathrm{Cr}\left(10^{3} \mathrm{psu} \mathrm{m}^{3} \mathrm{día}^{-1}\right)$ & 0.47 & 0.33 \\
$\mathrm{~F}$ & 0.15 & 0.05 \\
$\mathrm{~g} * \mathrm{f}$ & 0.16 & 0.20 \\
$\mathrm{~T}(\operatorname{años})$ & 469 & 375 \\
$\mathrm{Tr} / \mathrm{Tm}($ años $)$ & 6.25 & 5
\end{tabular}

superficie es transportado del fondo en forma particulada "g", y en la época lluviosa esta fracción es de $98 \%$, lo que coincide con el consecuente incremento en los flujos estacionales de surgencia (VmCp) y escorrentía ( $\mathrm{VrCr}$ ) que se observan en las correspondientes proporciones $\mathrm{Cp} / \mathrm{Cr}$ y $\mathrm{Cs} / \mathrm{Cr}$ de cada época climática.

La fracción de partículas que no se disuelven y luego sedimentan es de $15 \%$ en época seca, disminuyendo a $5 \%$ en época lluviosa, estas fracciones coinciden con las proporciones de agua aportadas por los ríos Tempisque y Tárcoles al sistema. Durante la época seca $\mathrm{Cr}=$ $23.4 \times 10^{3} \% \mathrm{~m}^{3} \mathrm{dí}^{-1}$ y $\mathrm{Cr}+\mathrm{Cm}+\mathrm{Cs}=101.3$ $\% \mathrm{~m}^{3}$ día $^{-1}$, la relación entre ambas indica que durante la época lluviosa el factor de dilución incrementa en un $23 \%$.

La fracción que se mantiene recirculando y que se pierde durante los ciclos de mezcla entre la zona profunda y la superficial, en la época seca corresponde a un $16 \%$, valor muy similar al que se observa durante el invierno, lo que da una idea del carácter conservativo del $\mathrm{NaCl}$ como principal componente de la salinidad. Smith y Crossland (1999) estiman que en promedio cada 41 días se produce una renovación total del agua del Golfo de Nicoya, lo que permitió calcular que su ciclo de mezcla vertical es de aproximadamente 75 años (cada 75 años un constituyente llega a la superficie proveniente del fondo). Por tanto, si el Golfo se mantuviera bajo las condiciones de época seca le tomaría al $\mathrm{NaCl} 469$ años depositarse en el fondo permanentemente y si tuviese condiciones lluviosas le tomaría 375 años, ello probablemente producto del incremento en los patrones de surgencia durante esta época.

\section{Modelo de Broecker y Peng aplicado a dos elementos biolimitantes}

Modelo para el balance de fosfato en el Golfo de Nicoya: El modelo se generó a partir de los estimados de FID obtenidos a través de los trabajos desarrollados por Cháves y Birkicht (1996), Krees et al. (1997), Smith y Crossland (1999), y Lachniet y Patterson (2002) en el Golfo de Nicoya.

De acuerdo con el modelo presentado se establece un flujo neto de fósforo que es exportado directamente por este ecosistema estuarino, el cual es casi un orden de magnitud mayor en la época lluviosa que en la época seca (Fig. 2). La diferencia en la producción de FID entre lo que se aporta (Cr) y lo que el Golfo de Nicoya exporta $(\mathrm{Cm})$ presenta un patrón similar. Los estimados de $\mathrm{Cr}$ son de $27 \times 10^{3} \mathrm{~mol}$ día ${ }^{-1}$ en la época seca y de $207 \times 10^{3} \mathrm{~mol} \mathrm{día}^{-1}$ en la época lluviosa. Estos valores coinciden con la tasa de liberación de fósforo inorgánico disuelto: $0.3 \times 10^{3} \mathrm{~mol} \mathrm{día}^{-1}$ en época seca y 0.4 x $10^{3} \mathrm{~mol} \mathrm{día}^{-1}$ en la época lluviosa, para una tasa promedio anual de cerca de $0.25 \times 10^{3} \mathrm{~mol}$ día ${ }^{-1}$ (Cuadro 3).

De acuerdo con el modelo durante la época seca, principalmente el aporte de fósforo por $\mathrm{VrCr}$ no es detectable, por tanto se asume que los niveles de producción basados en la necesidad de este nutrimento biolimitado dependen principalmente de la liberación biológica o del aporte de aguas ubicadas bajo la termoclina, el cual se estima para la época seca en $32040 \times 10^{3} \mathrm{~mol} \mathrm{día}^{-1}$. Durante la época lluviosa el incremento en los aportes de nutrimentos por lixiviación es enorme, por lo que es probable que la mayor parte del 


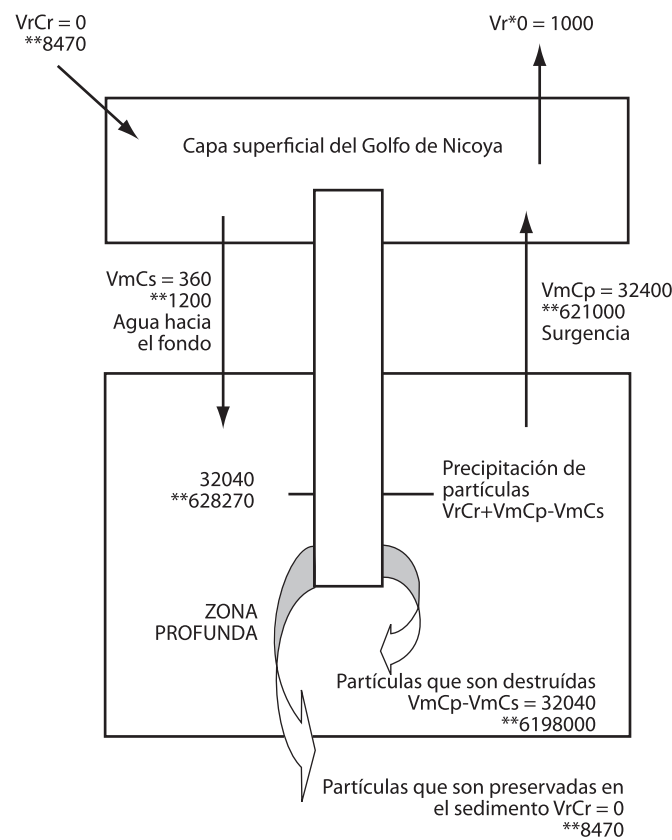

Fig. 2. Modelo de Broecker y Penn aplicado a la dinámica del flujo de fósforo inorgánico disuelto en el Golfo de Nicoya, Costa Rica, durante la época seca y la época lluviosa(**). Los flujos de FID se expresan en $10^{3}$ moles día ${ }^{-1}$

Fig. 2. Broecker \& Penn model applied to the fulx of dissolved inorganic phosphorus in the Gulf of Nicoya, Costa Rica, during the dry and rainy $(* *)$ seasons. DIP flow in $10^{3} \mathrm{~mol} \mathrm{day}{ }^{-1}$

\section{CUADRO 3}

Variación de los parámetros de Fosforo Inorgánico Disuelto (FID) según modelo de Broecker y Penn para la estación seca y la lluviosa, Golfo de Nicoya, Costa Rica

TABLE 3

Variation of Broecker \& Penn model's dissolved inorganic phosphorus (DIP) parameters for the dry and rainy seasons, Gulf of Nicoya, Costa Rica

Parámetro época seca época lluviosa

$\begin{array}{lcc}\text { Vr FID }\left(10^{3} \mathrm{~m}^{3} \text { día }^{-1}\right) & 1675 & 25300 \\ \text { Cr }\left(\times 10^{3} \text { moles día }^{-1}\right) & 0 & 0.7 \\ \text { Vm }\left(10^{3} \mathrm{~m}^{3} \mathrm{dí}^{-1}\right)^{*} & 1200 & 3000 \\ \text { Cp }\left(\times 10^{3} \text { moles día }\right. & \\ \text { Cs }\left(\times 10^{3} \text { moles día }^{-1}\right) & 27 & 207 \\ & 0.3 & 0.4\end{array}$

fósforo que llega a depositarse al sedimento se almacena, siendo en la época seca vital para el mantenimiento de los niveles de productividad primaria, a través del proceso de transporte por surgencia, el cual bajo una concepción temporal es analizado por Brenes et al. (1996). La introducción de nutrimentos al Golfo, producto del incremento en los niveles de lixiviación durante la época lluviosa, parece explicar el aumento en la concentración de clorofila $a$ (Krees et al. 1997).

Al no detectarse FID proveniente de la lixiviación durante la época seca los estimados se hacen cero. Ello se refleja al analizarse los valores de "g", tanto en la época seca como en la lluviosa parece detectarse una completa utilización del fósforo, lo que coincide con los informes que ubican al Golfo de Nicoya como un sistema oligotrófico con tendencias eutróficas durante la época lluviosa (Peterson 1958).

Modelo para el balance de NID en el Golfo de Nicoya: El Golfo de Nicoya es una fuente neta de NID con una producción total estimada en $53 \times 10^{3} \mathrm{~mol} \mathrm{día}^{-1}$ en la época seca y una mucho mayor de $3058 \times 10^{3} \mathrm{~mol} \mathrm{día}^{-1}$ en la época lluviosa (Smith y Crossland 1999).

En los Cuadros 4 y 5 se observan los estimados de los parámetros del modelo de Broecker y Penn aplicado para el flujo de NID en el Golfo de Nicoya, para la época seca y la lluviosa.

En condiciones de época seca, el $86 \%$ del nitrógeno que alcanza la superficie es transportado desde el fondo en forma particulada, y en época lluviosa casi el $100 \%$, lo que se refleja en las proporciones de NID provenientes del fondo en relación conl que proviene por lixiviación de los ríos. En la época seca esta tasa es de $29 \times 10^{3} \mathrm{~mol} \mathrm{día}^{-1}$ y en época lluviosa incrementa a $46.89 \times 10^{3} \mathrm{~mol} \mathrm{día}^{-1}$. La fracción de partículas que no se disuelven y luego sedimentan es muy similar, lo cual es coincidente con el carácter de nutriente biolimitante del NID (Cuadro 6). Resultados coincidentes se observan con la fracción que se mantiene recirculando y luego se sedimenta (g·f) (Fig. 3). 
CUADRO 4

Dinámica de flujos de FIP estimados por el modelo de Broecker y Penn para el Golfo de Nicoya, durante la época seca y la lluviosa

TABLE 4

DIP flow dynamics estimated by Broecker \& Penn model's in the Gulf of Nicoya, during the dry and rainy seasons

Parámetro

g

$\mathrm{Cp} / \mathrm{Cr}\left(10^{3}\right.$ moles día $\left.{ }^{-1}\right)$

$\mathrm{Cs} / \mathrm{Cr}\left(10^{3}\right.$ moles día $\left.^{-1}\right)$

f

$\mathrm{g} * \mathrm{f}$

$\mathrm{T}(\mathrm{años})$

$\operatorname{Tr} / \operatorname{Tm}($ años $)$

$\begin{array}{cc}\text { Época seca } & \text { Época lluviosa } \\ 0.99 & 1.00 \\ 0 & 295.7 \\ 0 & 0.57 \\ 0 & 0.11 \\ 0 & 0.18 \\ 0 & 417 \\ 0 & 5.6\end{array}$

\section{CUADRO 5}

Variación de los parámetros del modelo de Broecker y Penn obtenidos para la estación seca y la lluviosa, para el Nitrógeno Inorgánico Disuelto (NID) en el Golfo de Nicoya, Costa Rica

TABLE 5

Variation of Broecker \& Penn model's dissolved inorganic nitrogen (DIN) parameters for the dry and rainy seasons; Gulf of Nicoya, Costa Rica

$\begin{array}{ccc}\text { Parámetro } & \text { Época seca } & \text { Época lluviosa } \\ \text { Vr NIDr }\left(10^{3} \mathrm{~m}^{3} \mathrm{dí}^{-1}\right) & 1675 & 25300 \\ \text { Cr }\left(\times 10^{3} \mathrm{moles} \mathrm{día}^{-1}\right) & 3 & 65 \\ \text { Vm }\left(10^{3} \mathrm{~m}^{3} \text { día }^{-1}\right)^{*} & 1200 & 3000 \\ \text { Cp }\left(\times 10^{3} \text { moles día }^{-1}\right) & 87 & 3048 \\ \text { Cs }\left(\times 10^{3} \text { moles día }{ }^{-1}\right) & 12 & 30\end{array}$

En cuanto al tiempo de permanencia en el fondo por épocas climáticas, queda claro que si el Golfo exhibiera condiciones de época seca de forma permanente la permanencia del NID sería mucho mayor, resultados que coinciden con los niveles de productividad primaria detectados por épocas en el Golfo de Nicoya.

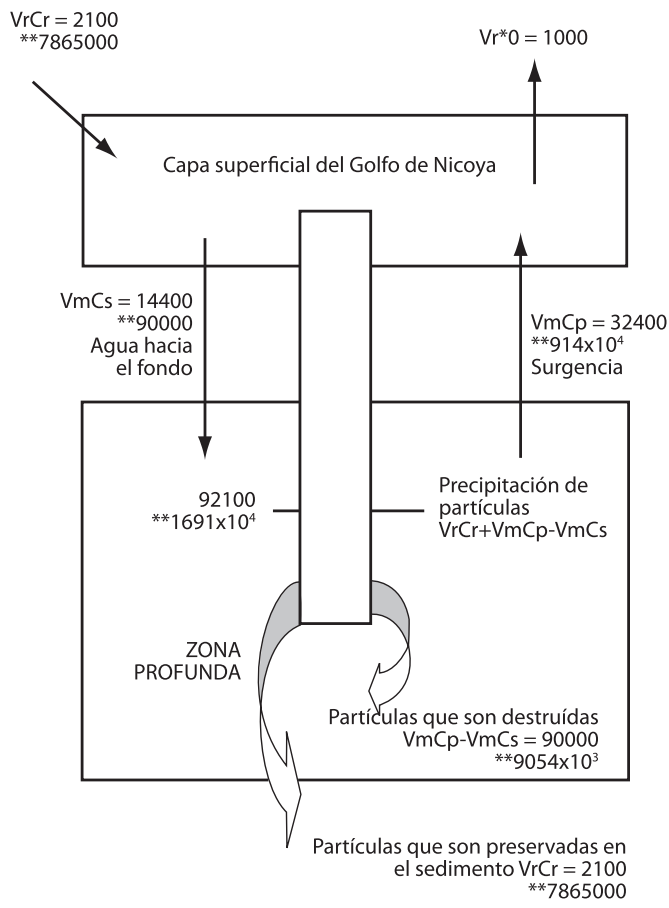

Fig. 3. Modelo de Broecker y Penn aplicado a la dinámica del flujo de nitrógeno inorgánico disuelto en el Golfo de Nicoya, Costa Rica, durante la época seca y la época lluviosa(**). Los flujos de NID se expresan en $10^{3}$ moles día ${ }^{-1}$ Fig. 3. Broecker \& Penn model for dissolved inorganic nitrogen in the Gulf of Nicoya, Costa Rica, during the dry and rainy $(* *)$ seasons. DIN flow in $10^{3} \mathrm{~mol} \mathrm{day}^{-1}$

\section{DISCUSIÓN}

Las partículas disueltas en las aguas del Golfo de Nicoya pueden ser removidas e integradas por diversos mecanismos, tal como se ha expresado en este modelo biogeoquímico, siendo el balance entre ellos lo que determina su composición y las tasas de intercambio de los elementos. Al considerar que el tiempo de mezcla del Golfo de Nicoya es de 75 años, sería factible especular que las variaciones en la concentración de los elementos biolimitantes ocurren cerca de su origen, en la desembocadura de los ríos Tempisque y Tárcoles y donde la 
CUADRO 6

Dinámica de flujos de NID estimados por el modelo de Broecker y Penn para el Golfo de Nicoya, durante la época seca y la lluviosa

TABLE 6

NID flow dynamics estimated by Broecker \& Penn model's for the Gulf of Nicoya, dry and rainy seasons

\begin{tabular}{lcc}
\multicolumn{1}{c}{ Parámetro } & Época seca & Época lluviosa \\
$\mathrm{G}$ & 0.86 & 0.99 \\
$\mathrm{Cp} / \mathrm{Cr}\left(10^{3} \mathrm{moles} \mathrm{día}^{-1}\right)$ & 29 & 46.89 \\
$\mathrm{Cs} / \mathrm{Cr}\left(10^{3} \mathrm{moles} \mathrm{día}^{-1}\right)$ & 4 & 0.46 \\
$\mathrm{~F}$ & 0.01 & 0.02 \\
$\mathrm{~g} * \mathrm{f}$ & 0.02 & 0.46 \\
$\mathrm{~T}(\mathrm{años})$ & 3750 & 163 \\
$\mathrm{Tr} / \mathrm{Tm}($ años $)$ & 50 & 2.17
\end{tabular}

precipitación y las variaciones en los niveles de evaporación hacen que el contenido salino también varíe significativamente

Al relacionar los ciclos químicos de los elementos biolimitantes con procesos de surgencia, lixiviación, deposición y reciclamiento dentro de un modelo biogeoquímico de dos capas como el propuesto para el Golfo de Nicoya, elementos como el $\mathrm{C}, \mathrm{N}, \mathrm{Na}, \mathrm{Cl}$ y $\mathrm{P}$, que se generan en la litosfera, la atmósfera y el océano varían estacionalmente en relación con los niveles de productividad primaria, procesos de desnitrificación (capa profunda) y nitrificación (capa superficial) del Golfo de Nicoya.

Los procesos biogeoquímicos juegan un papel importante en el ciclo de sedimentación y en los procesos de reciclamiento que se presentan en el Golfo de Nicoya, por lo que a raíz de los resultados en la aplicación de este modelo básico, se plantea el siguiente escenario: en el Golfo de Nicoya, los procesos biogeoquímicos se originan principalmente a partir de la radiación solar, la cual provee la energía necesaria para la generación de materia orgánica a partir de la fotosíntesis. Elementos biolimitantes como el nitrógeno y el fósforo al encontrarse en su forma química son termodinámicamente inestables, o sea, sus concentraciones son el reflejo del balance entre su continúa producción biológica (fotosíntesis) y su conversión hacia especies químicas más estables.

Para el caso del nitrógeno, su principal fuente es el aporte terrígeno a través de las desembocaduras del Tempisque y el Tárcoles, donde llega casi siempre en forma de iones nitrato, que presentan una alta variabilidad temporal y espacial producto de sus diversos estados de oxidación, ya que su química marina se encuentra ampliamente controlada por reacciones redox mediadas por fitoplancton y bacterias, que producen nitratos y nitritos para conformar parte de lo que se conoce como NID. Por tanto, es posible comprender la variabilidad temporal y espacial de la productividad primaria del Golfo de Nicoya (Peterson 1958, Bartels et al. 1983, Lizano y Vargas 1993, Cháves y Birkicht 1996, Brugnoli 1998, Smith y Crossland 1999, Calvo 2002).

En época seca, la disponibilidad de NID es $87 \times 10^{3} \mathrm{~mol} \mathrm{día}^{-1}$; mientras que en la época lluviosa incrementa a $3044 \times 10^{3} \mathrm{~mol} \mathrm{día}^{-1}$, resultados muy similares a los informados por Smith y Crossland (1999). Esto favorece el incremento en los niveles de productividad primaria durante la época lluviosa y por ende los procesos de desove de especies de ciclo de vida corto como la sardina, la anchoveta y el camarón (Bravo 1978, 1979, Carranza y Chacón 1979, Carranza 1985, Alfaro et al. 1993, Angulo 1993, Palacios 2003) y de ciclo de vida largo como el pargo y la corvina (Leventhal 1982, Gutiérrez 1990, Rojas 1994).

Cháves y Birkicht (1996) afirman que en el Golfo de Nicoya, los nutrientes biolimitantes presentan estratificación vertical, con concentraciones más altas en la parte profunda, bajo la termoclina, y que además coinciden con la propuesta de este modelo al otorgarle al aporte estacional de los ríos Tárcoles y Tempisque la mayor cuota de ingreso de estos nutrientes biolimitados. Sin embargo, ellos afirman que las mayores concentraciones espaciales de nitrógeno y fósforo se encontraron a la entrada del Golfo, atribuyéndose ello al aporte alóctono producido por el ingreso de la Corriente Ecuatorial Sub-Superficial (CESS) (Epifanio et al. 1983, Cháves y Birkicht 1996). 
Smith y Crossland (1999) afirman que el ingreso estacional de materia orgánica proveniente del río Tempisque y de los manglares aledaños aunado a los procesos de reciclamiento de nutrientes contribuyen a mantener las proporciones de los nutrientes biolimitados acorde con Redfield (1934), presentándose a su vez, cambios en su concentración entre la época seca y la lluviosa.

Cháves y Birkicht (1996), no obstante consideran que el probable efecto de desnitrificación causado por la baja concentración de oxígeno con que ingresa la CESS al Golfo de Nicoya, provoca variaciones en estas proporciones de los nutrientes.

Ciclos como los del carbono, el nitrógeno y el fósforo son críticamente dependientes de la Productividad Primaria Neta, o sea, de la fracción del carbono que es fotosintéticamente asimilable para otros niveles tróficos. Al establecer las variaciones en los niveles de nutrientes biolimitados con las de la Productividad Primaria en el Golfo de Nicoya, será posible correlacionarlos cualitativamente con procesos de circulación de corrientes y con procesos físicos de mesoescala, especialmente por su influencia con procesos de surgencia y re-suspensión, como agentes transportadores de nutrientes desde la zona profunda del Golfo de Nicoya e incluso desde la parte externa hacia la parte interna.

En conclusión se establece que la aplicación del modelo de Broecker y Penn, al analizar los flujos de agua y $\mathrm{NaCl}$ y de los dos nutrientes biolimitantes, coincide con los procesos biológicos estudiados e informados para el Golfo de Nicoya y podría ser una herramienta valiosa para la comprensión integral de un ecosistema tan complejo como el Golfo de Nicoya.

\section{RESUMEN}

De acuerdo con el "modelo de dos cajas de Broecker y Peng", se desarrolló un modelo de balance biogeoquímico del Golfo de Nicoya, considerando dos reservorios de nutrientes: aguas superficiales y aguas profundas. La zona de mezcla se ubicó en promedio a los $20 \mathrm{~m}$ de profundidad y se consideró que existe un balance entre las aguas superficiales que van hacia el fondo y un sistema de surgencia que lleva nutrientes y otros elementos químicos del fondo a la superficie. La principal fuente de nitrógeno, en forma de iones de nitrato, es el aporte terrígeno a través de las desembocaduras del Tempisque y el Tárcoles. El Golfo de Nicoya es una fuente neta de Nitrógeno Inorgánico Disuelto (NID) con una disponibilidad estimada en $87 \times 10^{3} \mathrm{~mol} \mathrm{día}^{-1}$ en época seca; mientras que en la época lluviosa incrementa a $3044 \times 10^{3} \mathrm{~mol} \mathrm{día}^{-1}$. Para el Fósforo Inorgánico Disuelto (FID) se estimó en $27 \mathrm{~mol}$ día $^{-1}$ en época seca y en $207 \mathrm{~mol} \mathrm{día}^{-1}$ en época lluviosa. La dinámica de estos nutrientes biolimitados, referida a las variaciones estacionales a través de sus aportes por lixiviación, coincide con los procesos biológicos informados para el Golfo de Nicoya, como variación en los niveles de productividad primaria, y épocas de madurez y reproducción de especies de ciclo de vida corto y largo.

Palabras clave: trópico, estuario, nutrientes, modelo biogeoquímico, Golfo de Nicoya, Costa Rica.

\section{REFERENCIAS}

Alfaro, J., J.A. Palacios, T. Aldave \& R. Angulo. 1993. Reproducción del camarón Penaeus occidentalis (Decapoda: Penaeidae) en el Golfo de Nicoya, Costa Rica. Rev. Biol. Trop. 41: 563-572.

Angulo, R. 1993. Crecimiento, mortalidad y madurez sexual de Penaeus stylirostris, Penaeus occidentalis y Trachipenaeus byrdi en el Golfo de Nicoya, Costa Rica. Tesis de Licenciatura, Universidad Nacional, Heredia, Costa Rica. 73 p.

Archer, D., G. Eshel, A. Winguth, W. Broecker, R. Pierrehumbert, M. Tobis \& R. Jacob. 2001. Atmospheric $\mathrm{pCO}_{2}$ sensitivity to the biological pump in the ocean, Global Biogeochem. Cycles 32: 63-75.

Bartels, C., K. Price, M. López \& W. Bussing. 1983. Occurrence, distribution and diversity of fishes in the Gulf of Nicoya, Costa Rica. Rev. Biol. Trop. 31: 75-101.

Bravo, E. 1978. Análisis sobre la pesca del camarón y sardina en la zona comprendida entre Negritos-Peñón y Puntarenas-Punta Gigante, periodo 1977. MAG: Dirección de Recursos Pesqueros y Vida Silvestre, San José, Costa Rica. 37 p.

Bravo, E. 1979. Características generales de los recursos camaroneros de Costa Rica. MAG: Dirección General de Recursos Pesqueros y Vida Silvestre, San José, Costa Rica. 27 p. 
Brenes, C., S. León \& G. Arroyo. 1996. Influence of coastal waters on some physical and chemical oceanographic characteristics of Gulf of Nicoya, Costa Rica. Top. Meteor. Oceanogr. 3: 65-72.

Brenes, C.L., J.E. Coen, D.B. Chelton, D.B. Enfield, S. León \& D. Ballestero. 2003. Wind driven upwelling in the Gulf of Nicoya, Costa Rica. Int. J. Remote Sens. 24: 1127-1133.

Broecker, W.J \& T.H. Peng. 1982. Internal cycling and throughput pathways from river mouth to sea floor, p. 690. In Tracer of the Sea. Chapter 1. LaMont-Boherty Geological Observatory, Columbia, EEUU.

Brugnoli, E. 1998. Factores abióticos y bióticos que regulan la estructura y dinámica de la comunidad fitoplanctónica en la zona de Punta Morales, Golfo de Nicoya. Tesis de Maestría, Universidad de Costa Rica, San José, Costa Rica. 73 p.

Brugnoli, E. \& A. Morales. 1999. Parámetros físico-químicos de la columna de agua, en la zona de Punta Morales, Golfo de Nicoya, durante un evento "La persona". Top. Meteor. Oceanog. 6: 52-57.

Calvo, V.E. 2002. Factores bióticos y abióticos relacionados con la distribución del dinoflagelado tóxico Pyrodinium bahamensis var. compressum (Plate 1906) en el Golfo de Nicoya, Costa Rica. Tesis de Licenciatura, Universidad Nacional, Heredia, Costa Rica. 58 p.

Carranza, F. 1985. Distribución y abundancia del recurso camarón blanco y alternativas de aprovechamiento mediante un "método de pesca artesanal" en el Golfo de Nicoya. Tesis de Maestría, UNAM, México DF, México. 60 p.

Carranza, F. \& A. Chacón. 1979. Estudios preliminares sobre la pesca del camarón blanco, café y rosado del litoral Pacífico costarricense. Ministerio de Agricultura y Ganadería, San José, Costa Rica. 45 p.

Cháves, J. \& M. Birkicht. 1996. Equatorial subsurface water and the nutrient seasonality distribution of the Gulf of Nicoya, Costa Rica. Rev. Biol. Trop. 44: 41-47.

Córdoba-Muñoz, M. del R. 1993. Productividad primaria en la columna de agua. Golfo de Nicoya, Costa Rica. Tesis de Maestría, Universidad de Costa Rica, San José, Costa Rica. 64 p.

Epifanio, C.E., D. Maurer \& A.I. Dittel. 1983. Seasonal changes in nutrients and dissolved oxygen in the Gulf of Nicoya, a tropical estuary in the Pacific coast of Central America. Hydrobiologia 101: 231-238.
Ganadesikan, A. 1999. A simple predictive model for the structure of the oceanic pycnocline. Science 283: 2077-2079.

Gutiérrez, R. 1990. Producción camaronera durante los últimos 15 años en el litoral pacífico de Costa Rica, p. 9. In Mem. Primer Sem. sobre la problemática pesquera en Costa Rica, 4-7 Dic. Puntarenas, Costa Rica.

Huwe, D. \& M. van der Ploeg. 1992. Biogeochemical Models in the Environmental Sciences the Dynamical System Paradigm and the Role of Simulation Modeling. HYLE-Int. J. Philos. Chem. 6: 117-142

Instituto Meteorológico Nacional. 2001. Reporte de precipitaciones, zona Pacífico Central. Instituto Meteorológico Nacional, San José, Costa Rica. 14 p.

Krees, N., S. Brenner, S. León \& C.L. Brenes. 1997 Ecological Assessment of the Gulf of Nicoya, Costa Rica, 50 p. In Final Report, Office of Agriculture \& Food Security Bureau for Global Programs, Field Support and Research US Agency for International Development, Washington DC, EEUU.

Lachniet, M.S. \& W.P. Patterson. 2002. Stable isotope values of Costa Rican waters. J. Hydrol. 260: 135 150.

Leventhal, K. 1982. Food habits of some commercial fish in the Gulf of Nicoya, Costa Rica. ACM, Tropical Field Research Prog, Costa Rica. 15 p.

Lizano, O. \& J. Vargas. 1993. Distribución espacio-temporal de la salinidad y la temperatura en la parte interna del Golfo de Nicoya. Tecnol. Marcha 12: 3-16.

Orr, J.C. \& J.L. Sarmiento. 1992. Potential of marine macroalgae as a sink for $\mathrm{CO}_{2}$ : constraints from a 3-D general circulation model of the global ocean. Water Air Soil Poll. 64: 405-421.

Palacios, J.A. 2003. Estado actual de los recursos pesqueros del litoral pacífico de Costa Rica: Cadena de productos hidrobiológicos, compromiso del sector pesca. Estación de Biología Marina, Universidad Nacional, Puntarenas, Costa Rica. 114 p.

Peterson, C.L. 1958. The physical oceanography of the Gulf of Nicoya, Costa Rica, a tropical estuary. Bull. Intern. Am. Trop. Tuna Comm. 3: 139-188.

Redfield, A.C. 1934. On the proportions of organic derivatives in seawater and their relation to the composition of plankton, p. 176-172. In R.J. Daniel (ed.). James Johnston Memorial Volume. Liverpool, Liverpool, Inglaterra. 
Rojas, M.J.R. 1994. Fecundidad. Épocas de reproducción y hábitos alimenticios del pargo mancha Lutjanus guttatus (Steindachner) (Pisces: Lutjanidae) en el Golfo de Nicoya, Costa Rica. Tesis de Licenciatura, Universidad Nacional, Heredia, Costa Rica. 89 p.

Sarmiento, J.L., \& J.R. Toggweiler. 1984. A new model for the role of the oceans in determining atmospheric $\mathrm{pCO}_{2}$. Nature 308: 621-624.

Schlitzer, R. 2000. Applying the Adjoint Method for Biogeochemical Modeling, p. 107-124. In P. Kasibhatla, M. Heimann, P. Rayner, N. Mahowald, R. Prinn \& D.E. Hartley (eds.) Inverse Methods in Global Biogeochemical Cycles. AGU Geophys. Monogr. series 114, Texas, EEUU.

Smith, C.J. \& C.J. Crossland. 1999. Biogeochemical budgeting of the Gulf of Nicoya. LOICZ Proced. 36: 346-349.

Valdes, J., C.L. Brenes, E. Solis \& M. Mendelewicz. 1987. Propiedades físico químicas de las aguas del Golfo de Nicoya, Costa Rica. Ing. Cienc. Quim. 11: 21-25.

Voorhis, A., E. Epifanio, D. Maurer, A. Dittel \& J. Vargas. 1983. The estuarine character of the Gulf of Nicoya, an embayment on the Pacific coast of Central America. Hydrobiologia 99: 225-237. 